\title{
Evaluation of Rapid Progressors in HIV Infection as an Extreme Phenotype
}

\author{
Ashley D. Olson, MA, Marguerite Guiguet, PhD, † Robert Zangerle, $\$$ John Gill, MD, $\|$ \\ Santiago Perez-Hoyos, PhD, $\mid$ Sara Lodi, PhD, \# Jade Ghosn, PhD, ** Maria Dorrucci, PhD, †† \\ Anne Johnson, PhD, \$t Mette Sannes, PhD, $\S \S$ Santiago Moreno, PhD, || || and Kholoud Porter,* \\ for CASCADE Collaboration in EuroCoord
}

\begin{abstract}
Design: Rapid CD4 cell loss represents an HIV phenotype used to identify causal variants of accelerated disease progression. The optimal rate and threshold for identifying this extreme phenotype in recently infected individuals is unclear.
\end{abstract}

\begin{abstract}
Methods: Using a cohort of patients with known dates of HIV-1 seroconversion (SC), CASCADE (Concerted Action on SeroConversion on AIDS and Death in Europe), we identified proportions experiencing nadir CD4 cell levels within 1 year of SC, and assessed their mean AIDS-free survival time at 10-year follow-up and hazard of AIDS/death, compared with those whose CD4 remained $>500$ cells per cubic millimeter. Follow-up was censored at December 31, 1996 to avoid bias due to combination antiretroviral therapy initiation.
\end{abstract}

Results: Of 4876 individuals, $2.8 \%, 7.3 \%$, and $24.9 \%$ experienced $\geq 1$ CD4 $<100,200$, and 350 cells per cubic millimeter, respectively, within 1 year of SC. Minimum CD4 levels of 30, 166, 231, and 506 cells per cubic millimeter were experienced during this period by $1 \%, 5 \%, 10 \%$, and $50 \%$ of individuals, respectively. Mean ( $95 \%$ confidence interval) AIDS-free survival at 10 years follow-up was 2.9 (2.3 to 3.6), 5.5 (5.0 to 6.1), 6.7 (6.5 to 7.0), 7.4 (7.2 to 7.6), and 8.1 (7.9 to 8.3), for those

Received for publication February 12, 2014; accepted April 23, 2014.

From the *Medical Research Council Clinical Trials Unit, University College London, London, United Kingdom; †INSERM U943, Paris, France; †UPMC, Paris, France; §Innsbruck Medical University, Innsbruck, Austria; ||University of Calgary, Calgary, Alberta, Canada; đVall d’Hebrón Institut de Recerca (VHIR), Universitat Autònoma de Barcelona, Barcelona, Spain; \#Instituto de Salud Carlos III, Madrid, Spain; **Service de Médecine Interne et Maladies Infectieuses, CHU Bicêtre, Paris, France; $\dagger \dagger$ † Department of Infectious, Parasitic and Immune-mediated Diseases, Istituto Superiore di Sanità, Rome, Italy; \$\$Institute of Epidemiology and Health Care, University College London, London, United Kingdom; §§Ulleval University Hospital, Oslo, Norway; and \|\| Hospital Universitário Ramón y Cajal, Madrid, Spain.

Supported by European Union Seventh Framework Program (FP7/20072013) under EuroCoord grant agreement no. 260694.

The authors have no conflicts of interest to disclose.

For a complete list of CASCADE Collaboration in EuroCoord, see Appendix 1. Supplemental digital content is available for this article. Direct URL citations appear in the printed text and are provided in the HTML and PDF versions of this article on the journal's Web site (www.jaids.com).

This is an open access article distributed under the Creative Commons Attribution License, which permits unrestricted use, distribution, and reproduction in any medium, provided the original work is properly cited.

Correspondence to: Ashley D. Olson, Medical Research Council Clinical Trials Unit, University College London, Aviation House, 125 Kingsway, London WC2B 6NH, United Kingdom (e-mail: a.olson@ucl.ac.uk).

Copyright $(2014$ by Lippincott Williams \& Wilkins with minimum counts $\leq 100,100-200,200-350,350-500,>500$ cells per cubic millimeter, respectively. Using counts of $>500$ cells per cubic millimeter as reference, the hazard ratios (95\% confidence interval) of AIDS/death were 15.0 (11.9 to 18.9), 3.6 (2.9 to 4.5), 2.1 (1.8 to 2.4 ), and 1.5 (1.3 to 1.7$)$, respectively. The hazard ratio increased to 37.5 (26.5 to 53.1) when a minimum CD4 count $<100$ was confirmed within 1 year of SC.

Conclusion: At least $1 \mathrm{CD} 4 \leq 100$ cells per cubic millimeter within the first year of SC identifies a rare group of individuals at high risk of disease progression and could form the basis for defining the rapid progressor phenotype.

Key Words: HIV, rare phenotype, disease progression, genetics

(J Acquir Immune Defic Syndr 2014;67:15-21)

\section{INTRODUCTION}

Rapid HIV disease progression is an extreme HIV phenotype, although there is little consensus on a definition. Differences in HIV disease progression can be assessed by variability in biomarkers related to HIV disease, the 2 most common of which being CD4 and HIV-RNA. Variability in HIV-RNA, specifically low-circulating HIV-RNA, has defined those with slow disease progression, termed longterm nonprogressor or elite controller phenotypes, which are of particular importance to vaccine studies. ${ }^{1-10}$ Rapid progression, however, is equally important as it also contributes to our understanding of early risk factors of disease progression. This may in turn help optimize the frequency of clinical monitoring and antiretroviral therapy initiation.

In addition to the well-documented relationship between slow disease progression and low HIV-RNA, there is also known variation in CD4 levels at or shortly after seroconversion (SC). A number of studies to date have defined rapid progression based on various levels of immunosuppression, ${ }^{11-21}$ but it is not yet clear if this variability in CD4, 1 early measure or consecutive low CD4 measurements do, indeed, constitute rapid progression and how this rare phenotype should be defined.

The CASCADE (Concerted Action on SeroConversion on AIDS and Death in Europe) Collaboration, of HIVpositive individuals followed-up since HIV SC offers a unique opportunity to evaluate HIV rapid progression. Using data from CASCADE, we aim to document low CD4 near SC and examine HIV rapid disease progression. This work provides 
the basis for choosing a definition appropriate to the objectives of future research on this extreme phenotype.

\section{METHODS}

\section{Study Population}

We used data from the CASCADE 2011 data release in EuroCoord (www.EuroCoord.net), which consists of 25,629 seroconverters from 28 cohorts across Europe, Canada, Australia, and sub-Saharan Africa. ${ }^{22}$ Date of HIV SC were estimated by various methods, most commonly as the midpoint between the last documented HIV-negative and the first positive HIV antibody test dates with an interval of $<3$ years between the 2 test dates $(85 \%)$. For the remainder, date of SC was estimated through laboratory evidence of SC (polymerase chain reaction positivity in the absence of HIV antibodies or antigen positivity with fewer than 4 bands on Western blot) (13\%), or as the date of a SC illness $(2 \%)$ with both an earlier documented negative and a later positive HIV test not more than 3 years apart. All cohorts contributing data to CASCADE received approval from their individual ethics review boards.

\section{Rapid Progression}

Because of the inconsistency of definitions described in the literature, ${ }^{11-21}$ with nadir CD4 cell counts ranging between 200 and 500 cells per cubic millimeter and follow-up ranging between 6 months and 8 years, we sought to identify those at highest risk of disease progression by severity of immunosuppression in early infection. We evaluated the frequency of low CD4 counts during the first year after SC and estimated the mean survival time at 10 years of follow-up and hazard of AIDS/death by nadir CD4 levels compared with individuals whose CD4 measurements remained above such levels during that period.

\section{Statistical Methods}

For all estimates, we considered only data from individuals who met the requirements for length of followup and minimum number of CD4 measurements. More specifically, only individuals with at least 1 CD4 count measured within the first year of SC could contribute information to assessing the risk associated with experiencing any specific nadir CD4 cell count within 1 year.

To provide estimates of the prevalence of low CD4 near SC, we calculated proportions experiencing various nadir CD4 levels within the first year of SC by mode of HIV transmission and age categories and plotted the cumulative proportion of individuals experiencing different nadir CD4 levels.

We used Cox proportional hazards models to estimate the relative hazard of AIDS/death among eligible individuals. As the nonproportionality assumption was not met in 3 of the 4 models, we used log-rank $P$ values. ${ }^{23}$ We estimated restricted mean AIDS-free survival times (the area under the length of AIDS-free survival curves) at 10 years of follow-up using clinically relevant CD4 categories $(<100,100-200,200-350,350-500$, and $>500$ cells/ $\mathrm{mm}^{3}$ ) using pseudovalues, as described previously. ${ }^{23,24}$ The follow-up time of 10 years was chosen as this is the median time to AIDS in the pre-combination antiretroviral therapy (cART) era for individuals infected between 25 and 35 years of age and was close to the last observed event time. ${ }^{25} \mathrm{We}$ also used fractional polynomials to explore the relationship of nadir CD4 measurements to the hazard of AIDS/death and restricted mean AIDS-free survival. ${ }^{26}$ For all models, we adjusted for the following potential confounders: sex, mode of HIV transmission, age at SC, and year at SC. Age and year of SC were modeled using restricted cubic splines with 3 knots. ${ }^{27}$ For all analyses, follow-up was censored at the earliest of AIDS or death date or on December 31, 1996 to avoid bias because of treatment initiation. AIDS was defined using the European case definition, which excludes CD4 $<200$ cells per cubic millimeter. ${ }^{28}$

In sensitivity analyses, we investigated the proportions experiencing nadir CD4 measurements within 6 months of SC

TABLE 1. Baseline Characteristics for (A) 4876 Individuals With $\geq 1$ CD4 Cell Measurement(s) Within 1 year of SC Included in Analysis and (B) 6084 Individuals Not Included in the Analysis but Seroconverting in the Pre-cART Era Using the CASCADE Data Set

\begin{tabular}{|c|c|c|}
\hline & $\mathbf{A}$ & B \\
\hline \multicolumn{3}{|l|}{ Risk category, n (\%) } \\
\hline MSM & $2564(53)$ & $2612(43)$ \\
\hline MSW & $1026(21)$ & $1741(29)$ \\
\hline IDU & $1085(22)$ & $1243(21)$ \\
\hline Other/unknown & $201(4)$ & $486(7)$ \\
\hline \multicolumn{3}{|l|}{ Sex, n (\%) } \\
\hline Male & $3798(78)$ & $4638(76)$ \\
\hline Female & $1078(22)$ & $1446(24)$ \\
\hline \multicolumn{3}{|l|}{ SC year } \\
\hline Median (IQR), yrs & 1992 (1989-1994) & $1991(1988-1994)$ \\
\hline \multicolumn{3}{|l|}{ SC age } \\
\hline Median (IQR), yrs & $29(25-35)$ & $28(24-33)$ \\
\hline$<20, \mathrm{n}(\%)$ & $239(5)$ & $454(7)$ \\
\hline$\geq 20-30, \mathrm{n}(\%)$ & $2481(51)$ & $3377(56)$ \\
\hline$\geq 30-40, \mathrm{n}(\%)$ & $1479(30)$ & $1613(27)$ \\
\hline$\geq 40, \mathrm{n}(\%)$ & $677(14)$ & $640(11)$ \\
\hline \multicolumn{3}{|l|}{ Geographical origin, $n(\%)$} \\
\hline Europe & $3652(78)$ & $4765(75)$ \\
\hline Africa & $117(3)$ & $170(2)$ \\
\hline Americas & $50(1)$ & $77(1)$ \\
\hline Unknown/Other & $1057(18)$ & $1072(22)$ \\
\hline $\begin{array}{l}\text { Time from SC to nadir CD4: } \\
\text { median (IQR), mo }\end{array}$ & $7.4(4.5-9.8)$ & - \\
\hline $\begin{array}{l}\text { Time from SC to first CD4: } \\
\text { median (IQR), mo }\end{array}$ & $5.2(3.0-7.9)$ & - \\
\hline $\begin{array}{l}\text { Number of CD4 counts: } \\
\text { median (IQR) }\end{array}$ & $2(1-2)$ & - \\
\hline Follow-up time: median (IQR) & $3.8(1.9-6.1)$ & - \\
\hline
\end{tabular}


and the hazard of AIDS/death observed by these minimum levels. We also investigated if confirmed CD4 measurements (ie, 2 counts) had an impact on the proportion, mean AIDSfree survival times, and hazard of AIDS/death within 6 months and 1 year of SC. Additionally, as the individuals in CASCADE are geographically diverse, we stratified all analysis by geographical origin. Analyses were conducted using Stata/IC 13.0.

\section{RESULTS}

\section{Baseline Characteristics}

Of 25,629 seroconverters, 20,753 were excluded for following reasons: 14,669 seroconverted after 1997, 6074 had no CD4 measurements within the first year of SC, 6 had an unknown AIDS date and 4 were $<15$ years of age at SC. Of the remaining 4876 individuals who were studied, 53\% were men infected through sex between men (men who have sex with men), $21 \%$ through heterosexual contact, $22 \%$ through injection drug use, and the remainder were hemophiliacs or with unknown risk categories. The majority (78\%) were male seroconverting at a median [interquartile range [IQR]) 29 (25-35) years old between 1982 and 1996. Median (IQR) time from SC to the lowest CD4 was 7.4 (4.5-9.8) months. Geographical origin was predominately European (78\%) with few individuals from Africa (3\%) and the Americas (1\%) (Table 1 ). HIV subtype was missing for $>80 \%$ of individuals in this analysis, but of those with known subtype, the data comprised mainly subtype B (>90\%).

Baseline characteristics of the 6084 individuals seroconverting in the pre-cART era excluded from this analysis and the sensitivity analysis were similar to the 4876 individuals included in this analysis (Table 1) (see Table S1, Supplemental Digital Content, http://links.lww.com/QAI/A542).

\section{CD4 Near SC}

Median (IQR) initial CD4 count during the first year of SC was 550 (384-726). A total of $138(2.8 \%), 356(7.3 \%)$, and $1213(24.9 \%)$ experienced at least 1 CD4 below 100, 200, and 350 cells per cubic millimeter, respectively, in the first year of SC (Fig. 1, Table 2). About 1\%, 5\%, 10\%, and $50 \%$ of individuals experienced at least 1 CD4 $<30,166$, 231, and 506 cells per cubic millimeter, respectively, within the first year of SC (Table 2). Higher CD4 cell levels were experienced by younger individuals and those infected through injection drug use (Table 2).

In sensitivity analyses, data were available from 2641 , 2825, and 894 individuals with a confirmed CD4 within 1 year of SC, at least $1 \mathrm{CD} 4$ within 6 months, and a confirmed CD4 within 6 months, respectively. Nadir CD4 percentiles remained qualitatively similar to those obtained from the main analysis (see Table S2, Supplemental Digital Content, http://links.lww.com/QAI/A542).
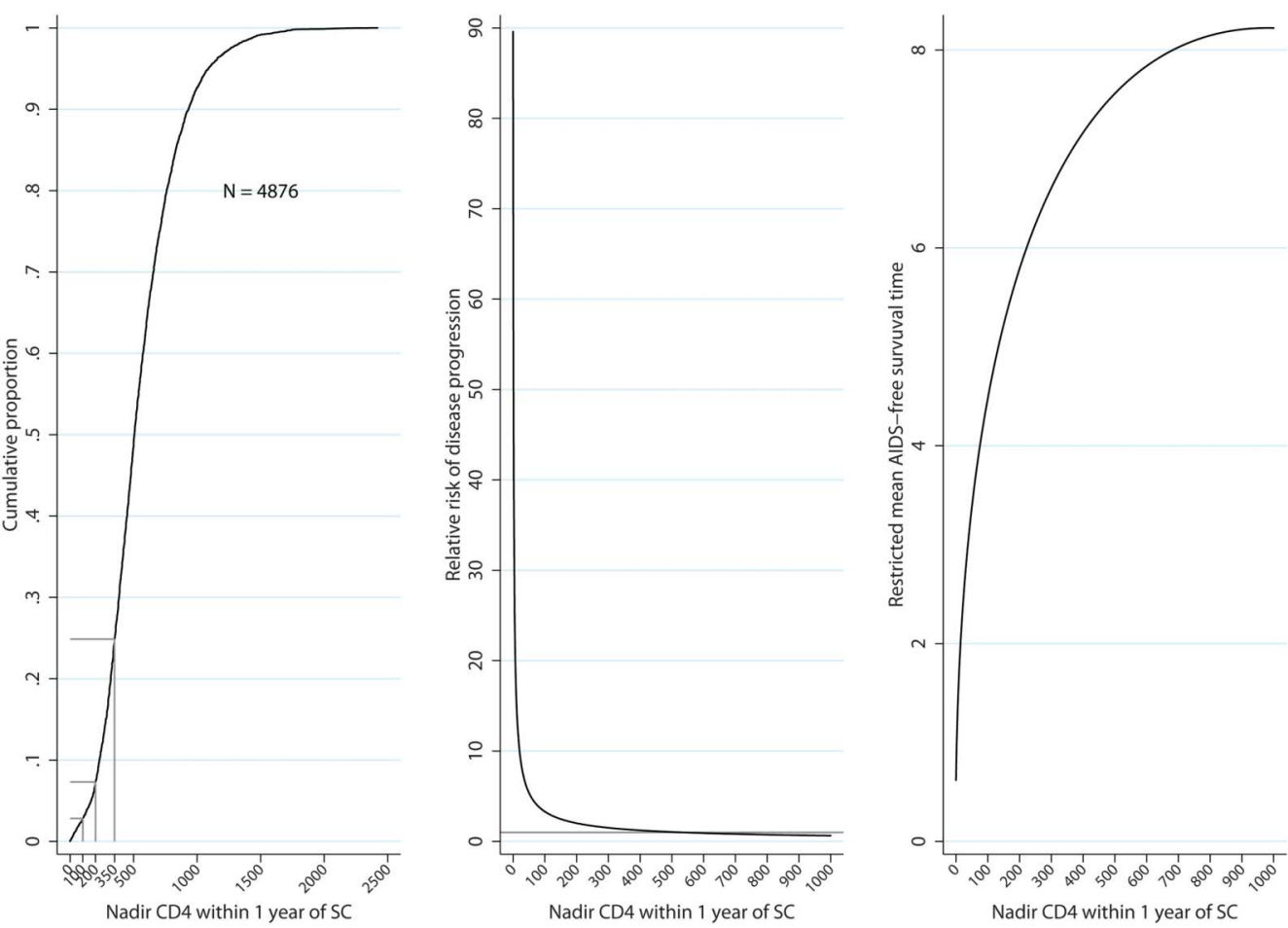

FIGURE 1. Cumulative proportions of nadir CD4 cell count (left hand panel), relative risk of AIDS/death compared with individuals whose CD4 counts remained at 500 cells per cubic millimeter (center panel), and mean AIDS-free survival time at 10 years follow-up (right hand panel) for individuals in CASCADE experiencing specific nadir levels within 1 year of SC during that period: all individuals seroconverted in the pre-CART era. 
TABLE 2. Minimum CD4 Percentiles Within 1 year of SC by HIV Risk Group and Age Categories in the Pre-cART Era Using the CASCADE Data Set

\begin{tabular}{|c|c|c|c|c|c|}
\hline & $<20$ & $\geq 20-30$ & $\geq 30-40$ & $\geq 40$ & Overall \\
\hline \multicolumn{6}{|l|}{$1 \%$} \\
\hline MSM & 165 & 49 & 27 & 30 & 35 \\
\hline IDU & 244 & 53 & 22 & 185 & 40 \\
\hline MSW & 154 & 19 & 27 & 23 & 27 \\
\hline OTH & - & 65 & 0 & 24 & 19 \\
\hline Overall & 156 & 49 & 24 & 24 & 30 \\
\hline \multicolumn{6}{|l|}{$5 \%$} \\
\hline MSM & 220 & 168 & 156 & 124 & 161 \\
\hline IDU & 318 & 210 & 141 & 240 & 200 \\
\hline MSW & 257 & 168 & 135 & 95 & 156 \\
\hline OTH & - & 136 & 48 & 64 & 112 \\
\hline Overall & 257 & 180 & 148 & 118 & 166 \\
\hline \multicolumn{6}{|l|}{$10 \%$} \\
\hline MSM & 270 & 237 & 223 & 213 & 224 \\
\hline IDU & 338 & 269 & 220 & 339 & 265 \\
\hline MSW & 300 & 255 & 220 & 190 & 225 \\
\hline OTH & - & 240 & 182 & 192 & 200 \\
\hline Overall & 307 & 250 & 220 & 204 & 231 \\
\hline \multicolumn{6}{|l|}{$25 \%$} \\
\hline MSM & 342 & 352 & 340 & 315 & 340 \\
\hline IDU & 439 & 425 & 343 & 364 & 405 \\
\hline MSW & 440 & 370 & 350 & 268 & 350 \\
\hline OTH & - & 404 & 289 & 328 & 318 \\
\hline Overall & 389 & 378 & 340 & 311 & 352 \\
\hline \multicolumn{6}{|l|}{$50 \%$} \\
\hline MSM & 450 & 500 & 487.5 & 441 & 484.5 \\
\hline IDU & 597 & 600 & 510 & 419.5 & 583 \\
\hline MSW & 560 & 519 & 491 & 430 & 501.5 \\
\hline ОТН & - & 546 & 500 & 474 & 502 \\
\hline Overall & 530 & 535 & 491 & 440 & 506 \\
\hline \multicolumn{6}{|l|}{$75 \%$} \\
\hline MSM & 638 & 676 & 650 & 614 & 654 \\
\hline IDU & 778 & 825 & 766 & 725 & 815 \\
\hline MSW & 840 & 732 & 722.5 & 603 & 751 \\
\hline OTH & 667 & 726 & 800 & 612 & 721 \\
\hline Overall & 717 & 736 & 680 & 612 & 705.5 \\
\hline \multicolumn{6}{|l|}{$90 \%$} \\
\hline MSM & - & 875 & 842 & 830 & 856 \\
\hline IDU & 947 & 1063 & 1045 & 1029 & 1057 \\
\hline MSW & 997 & 979 & 970 & 824 & 950 \\
\hline OTH & 842.5 & 977 & 1154 & 800 & 942 \\
\hline Overall & 947 & 966 & 907 & 830 & 933 \\
\hline \multicolumn{6}{|l|}{$100 \%$} \\
\hline MSM & 1250 & 1704 & 1875 & 1584 & 1875 \\
\hline IDU & 1984 & 2105 & 1744 & 2420 & 2420 \\
\hline MSW & 1453 & 2231 & 2156 & 1625 & 2231 \\
\hline OTH & - & 1704 & 2068 & 1357 & 2068 \\
\hline Overall & 1984 & 2231 & 2156 & 2420 & 2420 \\
\hline
\end{tabular}

IDU, injection drug users; MSM, men who have sex with men; MSW, heterosexual contact; OTH, hemophiliacs or unknown.

\section{Predicted Mean Survival Time at 10-Year Follow-Up}

The AIDS-free survival expectancy at 10 years follow-up significantly increased as nadir CD4 count measured within the first year of SC increased (Fig. 1, Table 3). Compared with individuals experiencing nadir CD4 counts $<100$ cells per cubic millimeter within the first year of SC, those with nadir CD4 of 100-200, 200-350, 350-500, and >500 have an increased AIDS-free survival expectancy of 2.6 (1.7-3.4), 3.8 (3.1-4.45), 4.5 (3.8-5.2), and 5.2 (4.5-5.5) years, respectively, during the first 10 years of HIV infection.

In a sensitivity analysis, the AIDS-free survival expectancy was qualitatively similar to results in the main analysis, increasing as nadir CD4 count and confirmed minimum CD4 measurements increased within 1 year and the 6 months of SC (Table 3). Predicted mean survival at 10 years follow-up was qualitatively similar when stratifying by geographical origin (data not shown).

\section{Risk of AIDS/Death by Nadir CD4}

The risk of AIDS/death increased as nadir CD4 count measured in the first year decreased. For individuals experiencing at least 1 count $<100$ cells per cubic millimeter, there was a 15 -fold increased risk of AIDS/death compared with those whose nadir CD4 count remained $>500$ cells per cubic millimeter. Hazard of AIDS/death was significantly higher for those with nadir counts $<500$ cells per cubic millimeter, (Fig. 1, Table 3).

In sensitivity analyses, the risk of AIDS/death was qualitatively similar to results in main analyses. As expected, however, the risk was greatly elevated for those experiencing confirmed counts, namely; hazard ratio (95\% confidence interval) 15.0 (11.9 to 18.9 ) vs. 37.5 (26.5 to 53.1) for CD4 $\leq 100$ cells per cubic millimeter and 3.6 (2.9 to 4.5 ) vs. 6.3 (4.5 to 8.8 ) for CD4 100-200 cells per cubic millimeter comparing a single minimum CD4 count with a confirmed CD4 count within 1 year of SC. This same pattern was observed when comparing nadir CD4 with a confirmed minimum CD4 count within 6 months of SC, (Table 3). There was a similar trend of higher risk of AIDS/ death for lower CD4 cell counts when stratifying by geographical origin (data not shown).

\section{DISCUSSION}

Individuals experiencing 1 or more CD4 cell count $\leq 100$ cells per cubic millimeter within the first year of SC provide a rare group (2.8\%) of HIV-positive individuals at the highest risk of disease progression with remarkably short mean AIDS-free survival of 2.9 years. These results suggest that CD4 monitoring close to SC may play an important role in identifying those at highest risk of progression. In addition to this, individuals with at least $1 \mathrm{CD} 4$ cell count $\leq 500$ cells per cubic millimeter are at an increased risk of AIDS/death compared with individuals whose CD4 remain above 500 cells per cubic millimeter.

We have shown that low CD4 cell counts $<100$ cells per cubic millimeter near $\mathrm{SC}$ is rare, but low $\mathrm{CD} 4$ near SC can 
TABLE 3. HR for Time to AIDS/Death by Nadir CD4 Measured Within 1 year of SC Using the CASCADE Data Set

\begin{tabular}{|c|c|c|c|c|c|c|}
\hline \multirow[b]{2}{*}{ CD4 Value, cells $/ \mathrm{mm}^{3}$} & \multicolumn{3}{|c|}{ Within 6 Months of SC } & \multicolumn{3}{|c|}{ Within 1 Year of SC } \\
\hline & N (Fail) & HR $(95 \% \text { CI })^{*}$ & $\begin{array}{c}\text { Mean AIDS-Free } \\
\text { Survival }(95 \% \text { CI) } \dagger\end{array}$ & N (Fail) & HR $(95 \% \text { CI })^{*}$ & $\begin{array}{c}\text { Mean AIDS-Free } \\
\text { Survival }(95 \% \text { CI }) \dagger\end{array}$ \\
\hline \multicolumn{7}{|l|}{ Nadir CD4 } \\
\hline$\leq 100$ & $58(37)$ & $12.8(9.0$ to 18.2$) \div$ & $3.1(2.1$ to 4.1$)$ & $138(94)$ & $15.0(11.9$ to 18.9$) \ddagger$ & $2.9(2.3$ to 3.6$)$ \\
\hline $100-200$ & $97(40)$ & $2.9(2.1$ to 4.1$)+\frac{t}{t}$ & $5.6(4.7$ to 6.5$) \S$ & $218(91)$ & $3.6(2.9$ to 4.5$) \ddagger$ & $5.5(5.0$ to 6.1$) \S$ \\
\hline $200-350$ & 409 (134) & $2.3(1.9$ to 2.8$) \frac{t}{t}$ & $6.3(5.9$ to 6.6$) \S$ & 857 (267) & $2.1(1.8$ to 2.4$) \ddagger$ & $6.7(6.5$ to 7.0$) \S$ \\
\hline $350-500$ & $607(180)$ & $1.6(1.3$ to 1.9$) \ddagger$ & $7.0(6.7$ to 7.3$) \S$ & $1185(316)$ & $1.5(1.3$ to 1.7$) \ddagger$ & $7.4(7.2$ to 7.6$) \S$ \\
\hline$>500$ & $1654(359)$ & $1 \div$ & $8.0(7.8$ to 8.1$) \S$ & $2478(501)$ & $1 \ddagger$ & $8.1(7.9$ to 8.3$) \S$ \\
\hline \multicolumn{7}{|l|}{ Confirmed CD4 } \\
\hline$\leq 100$ & $14(10)$ & 49.3 (23.4 to 104.2$)$ & $2.1(0.4$ to 3.7$)$ & $64(51)$ & 37.5 (26.5 to 53.1$) \ddagger$ & $1.8(1.1$ to 2.6$)$ \\
\hline $100-200$ & $33(17)$ & $7.4(4.2$ to 13.0$)$ & $3.6(2.3$ to 5.0$)$ & $92(40)$ & $6.3(4.5$ to 8.8$) \ddagger$ & $4.9(4.0$ to 5.7$) \S$ \\
\hline $200-350$ & $118(36)$ & $2.5(1.7$ to 3.7$)$ & $5.6(4.9$ to 6.4$) \S$ & $342(106)$ & $2.4(1.9$ to 3.0$) \dagger$ & $6.3(5.9$ to 6.7$) \S$ \\
\hline $350-500$ & $186(61)$ & $1.6(1.2$ to 2.3$)$ & $6.1(5.5$ to 6.7$) \S$ & $577(171)$ & $1.8(1.5$ to 2.1$) \ddagger$ & $6.8(6.4$ to 7.1$) \S$ \\
\hline$>500$ & $543(130)$ & 1 & 7.3 (6.9 to 7.7$) \S$ & $1566(344)$ & $1 \ddagger$ & $7.9(7.7$ to 8.1$) \S$ \\
\hline
\end{tabular}

*Adjusted for sex, risk group, SC age, SC year, log-rank test $P<0.001$.

$\dagger$ Mean AIDS-free survival at 10 years of follow-up.

†Cox proportional hazards $P<0.05$.

§Statistically greater than $\leq 100$ cells per cubic millimeter category $(P<0.001)$.

$\mathrm{CI}$, confidence interval; HR, hazard ratio.

have other research implications. Our definition can be useful for researching extreme phenotypes, particularly for genetic studies aiming to identify rare causal variants by looking at extreme ends of HIV disease progression. ${ }^{29,30}$ In addition to genetic implications, low CD4 near SC can have impact on HIV incidence measurements. HIV incidence measures such as The Recent Incidence Testing Algorithm aim to identify individuals infected within 4-6 months of sampling but exclude individuals who have AIDS, on ART or are identified with low CD4 as these individuals have been shown to be misclassified as recently infected. ${ }^{31}$ Our study suggests that up to $5 \%$ of the HIV-positive population tested in the first 6 months of SC will have a CD4 below 200 and thus would be misclassified as longstanding infection according to The Recent Incidence Testing Algorithm. These results suggest the need for an incidence estimate correction factor to account for low CD4 cell counts near SC.

Individuals experiencing confirmed low CD4 measurements $<200$ cells per cubic millimeter had more than a 2-fold increased risk of AIDS/death compared with minimum CD4 measurement alone. Although this may suggest that a confirmatory CD4 has a higher prognostic value of disease progression than a single CD4 alone, it is unusual for individuals to have a confirmed CD4 so close to $\mathrm{SC}$, shown by our reduced numbers for this population. We were able to analyze repeated low CD4 measurements because these data were restricted to the pre-cART era; however, it is unlikely that in the cART era naive low confirmed CD4 measurements would be available, as all individuals with 1 CD4 $<200$ cells per cubic millimeter are recommended to be on treatment.

Subtype was missing for $>80 \%$ of individuals in this analysis, and comprised mainly subtype B (90\%), which compared with other HIV subtypes, has previously been shown to have different rates of CD4 cell levels near SC and CD4 rates of decline suggesting these results may not be generalizable to other HIV subtypes. ${ }^{32}$ We stratified the analysis by geographical origin and the same trend of higher risk of disease progression with lower CD4 cell counts was observed, suggesting these results are generalizable in different global epidemics.

Our study has several strengths. First, the availability of SC estimation is essential to identifying individuals with rapid disease progression. Without laboratory evidence of SC, individuals entering care with low CD4 would be termed late presenters instead of rapid progressors. ${ }^{33}$ Second, the availability of data in an era when ART was not used early in the course of disease allowed us to assess rapid progression without the interaction of ART on disease progression. In the cART era, individuals with CD4 $<350$ cells per cubic millimeter would be on cART and the impact of low CD $4<100$ cells per cubic millimeter near SC would not be fully understood. Finally, the large sample size of our cohort allows us to compare between different possible combinations of this rare phenotype.

Our study has limitations. SC illness and HIV test intervals $<31$ days have been shown to be associated with faster disease progression, ${ }^{34,35}$ suggesting our proportion and risk estimates could be overinflated because of the increased likelihood of individuals seeking care when experiencing SC illness, although the midpoint method of estimating SC was used for $85 \%$ of seroconverters. We were unable to test if rapid progressors are more likely to report SC illness, as this is unknown in $>70 \%$ of the CASCADE data set. However, among 1481 individuals in our study with known SC illness status, $>50 \%$ of individuals reported of SC illness with $\mathrm{CD} 4$ count $<350$ cells per cubic millimeter, where $<50 \%$ of individuals reported no $\mathrm{SC}$ illness in those with a CD4 count $\geq 350$ cells per cubic millimeter (data not shown). Although our study only 
investigates seroconverters, it has been shown that HIV progression, in particular CD4 decline, among seroconverters is similar to that of the general HIV-positive population suggesting our results are generalizable to the HIV-positive population. ${ }^{36}$

In conclusion, individuals with at least 1 CD4 $\leq 100$ cells per cubic millimeter in the first year of SC are a rare and extreme group who are at a very high risk of rapid disease progression. Given that the HIV test intervals in this study are consistent with HIV testing guidelines, ${ }^{37-40}$ our study allows clinicians to identify individuals at risk of progression at an early stage for whom immediate initiation of therapy may be indicated. This study has also helps to identify an extreme HIV phenotype that increases power to detect rare variants in causal viral and host genetics of rapid HIV disease progression. This may, in turn, lead to targeted treatments for individuals at the greatest risk of progression. We suggest future research use at least $1 \mathrm{CD} 4 \leq 100$ cells per cubic millimeter within 1 year of SC as a definition for rapid progression.

\section{REFERENCES}

1. Deeks SG, Walker BD. Human immunodeficiency virus controllers: mechanisms of durable virus control in the absence of antiretroviral therapy. Immunity. 2007;27:406-416.

2. Eriksson EM, Milush JM, Ho EL, et al. Expansion of CD8+ T cells lacking Sema4D/CD100 during HIV-1 infection identifies a subset of T cells with decreased functional capacity. Blood. 2012;119:745-755.

3. Lambotte O, Boufassa F, Madec Y, et al. HIV controllers: a homogeneous group of HIV-1-infected patients with spontaneous control of viral replication. Clin Infect Dis. 2005;41:1053-1056.

4. Lopez M, Soriano V, Peris-Pertusa A, et al. Elite controllers display higher activation on central memory CD8 T cells than HIV patients successfully on HAART. AIDS Res Hum Retroviruses. 2011;27: $157-165$

5. Okulicz JF, Grandits GA, Weintrob AC, et al. CD4 T cell count reconstitution in HIV controllers after highly active antiretroviral therapy. Clin Infect Dis. 2010;50:1187-1191

6. Okulicz JF, Marconi VC, Landrum ML, et al. Clinical outcomes of elite controllers, viremic controllers, and long-term nonprogressors in the US Department of Defense HIV natural history study. J Infect Dis. 2009;200: 1714-1723

7. Owen RE, Heitman JW, Hirschkorn DF, et al. HIV+ elite controllers have low HIV-specific T-cell activation yet maintain strong, polyfunctional T-cell responses. AIDS. 2010;24:1095-1105.

8. Pereyra F, Addo MM, Kaufmann DE, et al. Genetic and immunologic heterogeneity among persons who control HIV infection in the absence of therapy. J Infect Dis. 2008;197:563-571.

9. Saez-Cirion A, Hamimi C, Bergamaschi A, et al. Restriction of HIV-1 replication in macrophages and CD4+ $\mathrm{T}$ cells from HIV controllers. Blood. 2011;118:955-964.

10. Walker BD. Elite control of HIV Infection: implications for vaccines and treatment. Top HIV Med. 2007;15:134-136.

11. Goicoechea M, Smith D, May S, et al. Prevalence and T-cell phenotype of slow HIV disease progressors with robust HIV replication. J Acquir Immune Defic Syndr. 2009;52:299-301.

12. Papasteriades $\mathrm{CH}$, Economidou J, Pappas H, et al. HLA antigens as predictors of disease progression in HIV-infected haemophilia patients (a 22 years' follow up). Haemophilia. 2005;11:371-375.

13. Quinones-Mateu ME, Ball SC, Marozsan AJ, et al. A dual infection competition assay shows a correlation between ex vivo human immunodeficiency virus type 1 fitness and disease progression. $J$ Virol 2000;74:9222-9233.

14. Audige A, Taffe P, Rickenbach M, et al. Low postseroconversion CD4 count and rapid decrease of CD4 density identify HIV+ fast progressors. AIDS Res Hum Retroviruses. 2010;26:997-1005.
15. Campbell GR, Pasquier E, Watkins J, et al. The glutamine-rich region of the HIV-1 Tat protein is involved in T-cell apoptosis. J Biol Chem. 2004; 279:48197-48204.

16. Li M, Song R, Masciotra S, et al. Association of CCR5 human haplogroup E with rapid HIV type 1 disease progression. AIDS Res Hum Retroviruses. 2005;21:111-115.

17. Loke P, Favre D, Hunt PW, et al. Correlating cellular and molecular signatures of mucosal immunity that distinguish HIV controllers from noncontrollers. Blood. 2010;115:e20-32.

18. Lopez-Vazquez A, Mina-Blanco A, Martinez-Borra J, et al. Interaction between KIR3DL1 and HLA-B*57 supertype alleles influences the progression of HIV-1 infection in a Zambian population. Hum Immunol. 2005;66:285-289.

19. Mao Q, Ray SC, Laeyendecker O, et al. Human immunodeficiency virus seroconversion and evolution of the hepatitis $\mathrm{C}$ virus quasispecies. J Virol. 2001;75:3259-3267.

20. Masciotra S, Owen SM, Rudolph D, et al. Temporal relationship between V1V2 variation, macrophage replication, and coreceptor adaptation during HIV-1 disease progression. AIDS. 2002;16:1887-1898.

21. Whittall T, Peters B, Rahman D, et al. Immunogenic and tolerogenic signatures in human immunodeficiency virus (HIV)-infected controllers compared with progressors and a conversion strategy of virus control. Clin Exp Immunol. 2011;166:208-217.

22. de Wolf F, Sabin C, Kirk O, et al. Developing a multidisciplinary network for clinical research on HIV infection: the EuroCoord experience. Clin Invest. 2012;2:255-264.

23. Royston $\mathrm{P}$, Parmar MK. The use of restricted mean survival time to estimate the treatment effect in randomized clinical trials when the proportional hazards assumption is in doubt. Stat Med. 2011;30:2409-2421.

24. Andersen PK, Hansen MG, Klein JP. Regression analysis of restricted mean survival time based on pseudo-observations. Lifetime Data Anal. 2004; 10:335-350.

25. Time from HIV-1 seroconversion to AIDS and death before widespread use of highly-active antiretroviral therapy: a collaborative re-analysis. Collaborative Group on AIDS Incubation and HIV Survival including the CASCADE EU Concerted Action. Concerted Action on SeroConversion to AIDS and Death in Europe. Lancet. 2000;355:1131-1137.

26. Royston P, Ambler G, Sauerbrei W. The use of fractional polynomials to model continuous risk variables in epidemiology. Int J Epidemiol. 1999; 28:964-974.

27. Durrleman S, Simon R. Flexible regression models with cubic splines. Stat Med. 1989;8:551-561.

28. Park RA. European AIDS definition. Lancet. 1992;339:671.

29. Cirulli ET, Goldstein DB. Uncovering the roles of rare variants in common disease through whole-genome sequencing. Nat Rev Genet. 2010; 11:415-425.

30. Barnett IJ, Lee S, Lin X. Detecting rare variant effects using extreme phenotype sampling in sequencing association studies. Genet Epidemiol. 2013;37:142-151.

31. Murphy G, Parry JV. Assays for the detection of recent infections with human immunodeficiency virus type 1. Euro Surveill. 2008;13:8.

32. Touloumi G, Pantazis N, Pillay D, et al. Impact of HIV-1 subtype on CD4 count at HIV seroconversion, rate of decline, and viral load set point in European seroconverter cohorts. Clin Infect Dis. 2013;56:888-897.

33. Sabin CA, Smith CJ, Gumley H, et al. Late presenters in the era of highly active antiretroviral therapy: uptake of and responses to antiretroviral therapy. AIDS. 2004;18:2145-2151.

34. The relationships between the HIV test interval, demographic factors and HIV disease progression. Epidemiol Infect. 2001;127:91-100.

35. Lindback S, Brostrom C, Karlsson A, et al. Does symptomatic primary HIV-1 infection accelerate progression to CDC stage IV disease, CD4 count below $200 \times 10(6) / 1$, AIDS, and death from AIDS? BMJ. 1994;309: $1535-1537$.

36. Lodi S, Phillips A, Touloumi G, et al. CD4 decline in seroconverter and seroprevalent individuals in the precombination of antiretroviral therapy era. AIDS. 2010;24:2697-2704.

37. European Centre for Disease Prevention and Control. HIV Testing: Increasing Uptake and Effectiveness in the European Union. Stockholm, Sweden: EDCD; 2010

38. National Institute for Health and Clinical Excellence. Increasing the uptake of HIV testing among men who have sex with men (Guidance: PH34). London: National Institute for Health and Clinical Excellence; 2011. 
39. National Institute for Health and Clinical Excellence. Increasing the uptake of HIV testing among men who have sex with men (Guidance: PH33). London: National Institute for Health and Clinical Excellence; 2011.

40. Centers for Disease Control and Prevention. Revised recommendations for HIV testing of adults, adolescents, and pregnant women in health-care settings. MMWR Recomm Rep. 2006;55:7-13.

\section{APPENDIX 1}

CASCADE Steering Committee: Julia Del Amo (Chair), Laurence Meyer (Vice Chair), Heiner C. Bucher, Geneviève Chêne, Osamah Hamouda, Deenan Pillay, Maria Prins, Magda Rosinska, Caroline Sabin, Giota Touloumi.

CASCADE Coordinating Centre: Kholoud Porter (Project Leader), Ashley Olson, Kate Coughlin, Lorraine Fradette, Sarah Walker, Abdel Babiker.

CASCADE Clinical Advisory Board: Heiner C. Bucher, Andrea De Luca, Martin Fisher, Roberto Muga.

CASCADE Collaborators: Australia: PHAEDRA cohort (Tony Kelleher, David Cooper, Pat Gray, Robert Finlayson, Mark Bloch) Sydney AIDS Prospective Study and Sydney Primary HIV Infection cohort (Tony Kelleher, Tim Ramacciotti, Linda Gelgor, David Cooper, Don Smith); Austria: Austrian HIV Cohort Study (Robert Zangerle); Canada: South Alberta clinic (John Gill); Estonia Tartu Ülikool (Irja Lutsar); France: ANRS CO3 Aquitaine cohort (Geneviève Chêne, Francois Dabis, Rodolphe Thiebaut), ANRS CO4 French Hospital Database (Dominique Costagliola, Marguerite Guiguet), Lyon Primary Infection cohort (Philippe Vanhems), French ANRS CO6 PRIMO cohort (Marie-Laure Chaix, Jade Ghosn), ANRS CO2 SEROCO cohort (Laurence Meyer, Faroudy Boufassa); Germany: German HIV-1 seroconverter cohort (Osamah Hamouda, Claudia Kücherer, Barbara Bartmeyer); Greece: AMACS (Anastasia Antoniadou, Georgios Chrysos, Georgios L. Daikos); Greek Haemophilia cohort (Giota Touloumi, Nikos Pantazis, Olga Katsarou); Italy: Italian Seroconversion Study (Giovanni Rezza, Maria Dorrucci), ICONA cohort (Antonella d'Arminio Monforte, Andrea De Luca); Netherlands: Amsterdam Cohort Studies among homosexual men and drug users (Maria Prins, Ronald Geskus, Jannie van der Helm, Hanneke Schuitemaker); Norway: Oslo and Ulleval Hospital cohorts (Mette Sannes, Oddbjorn Brubakk, Anne-Marte Bakken Kran); Poland: National Institute of Hygiene (Magdalena Rosinska); Spain: Badalona IDU hospital cohort (Roberto Muga, Jordi Tor), Barcelona IDU Cohort (Patricia Garcia de Olalla, Joan Cayla), CoRIS-scr (Julia del Amo, Santiago Moreno, Susana Monge); Madrid cohort (Julia Del Amo, Jorge del Romero), Valencia IDU cohort (Santiago Pérez-Hoyos); Sweden: Swedish InfCare HIV Cohort, Sweden (Anders Sönnerborg); Switzerland: Swiss HIV Cohort Study (Heiner C. Bucher, Martin Rickenbach, Patrick Francioli); Ukraine: Perinatal Prevention of AIDS Initiative (Ruslan Malyuta); United Kingdom: Public Health England (Gary Murphy), Royal Free haemophilia cohort (Caroline Sabin), UK Register of HIV Seroconverters (Kholoud Porter, Anne Johnson, Andrew Phillips, Abdel Babiker), University College London (Deenan Pillay). African cohorts: Genital Shedding Study (US: Charles Morrison; Family Health International, Robert Salata, Case Western Reserve University, Uganda: Roy Mugerwa, Makerere University, Zimbabwe: Tsungai Chipato, University of Zimbabwe); Interna- tional AIDS Vaccine Initiative (IAVI) Early Infections Cohort (Kenya, Rwanda, South Africa, Uganda, Zambia: Pauli N. Amornkul, IAVI, USA; Jill Gilmour, IAVI, United Kingdom; Anatoli Kamali, Uganda Virus Research Institute/Medical Research Council Uganda; Etienne Karita, Projet San Francisco, Rwanda).

EuroCoord Executive Board: Julia del Amo, Instituto de Salud Carlos III, Spain; Geneviève Chêne, University of Bordeaux II, France; Dominique Costagliola, Institut National de la Santé et de la Recherche Médicale, France; Carlo Giaquinto, Fondazione PENTA, Italy; Jesper Grarup, Københavns Universitet, Denmark; Ole Kirk (Chair), Københavns Universitet, Denmark; Laurence Meyer, Institut National de la Santé et de la Recherche Médicale, France; Ashley Olson, University College London, United Kingdom; Alex Panteleev, St. Petersburg City AIDS Centre, Russian Federation; Lars Peters, Københavns Universitet, Denmark; Andrew Phillips, University College London, United Kingdom, Kholoud Porter, University College London, United Kingdom; Peter Reiss (Scientific Coordinator), Academic Medical Centre University of Amsterdam, Netherlands; Claire Thorne, University College London, United Kingdom.

EuroCoord Council of Partners: Jean-Pierre Aboulker, Institut National de la Santé et de la Recherche Médicale, France; Jan Albert, Karolinska Institute, Sweden; Silvia Asandi, Romanian Angel Appeal Foundation, Romania; Geneviève Chêne, University of Bordeaux II, France; Dominique Costagliola, INSERM, France; Antonella d'Arminio Monforte, ICoNA Foundation, Italy; Stéphane De Wit, St. Pierre University Hospital, Belgium; Peter Reiss, Stichting HIV Monitoring, Netherlands; Julia Del Amo, Instituto de Salud Carlos III, Spain; José Gatell (Chair), Fundació Privada Clínic per a la Recerca Bíomèdica, Spain; Carlo Giaquinto, Fondazione PENTA, Italy; Osamah Hamouda, Robert Koch Institut, Germany; Igor Karpov, University of Minsk, Belarus; Bruno Ledergerber, University of Zurich, Switzerland; Jens Lundgren, Københavns Universitet, Denmark; Ruslan Malyuta, Perinatal Prevention of AIDS Initiative, Ukraine; Claus Møller, Cadpeople A/S, Denmark; Kholoud Porter, University College London, United Kingdom; Maria Prins, Academic Medical Centre, Netherlands; Aza Rakhmanova, St. Petersburg City AIDS Centre, Russian Federation; Jürgen Rockstroh, University of Bonn, Germany; Magda Rosinska, National Institute of Public Health, National Institute of Hygiene, Poland; Manjinder Sandhu, Genome Research Limited; Claire Thorne, University College London, United Kingdom; Giota Touloumi, National and Kapodistrian University of Athens, Greece; Alain Volny Anne, European AIDS Treatment Group, France.

EuroCoord External Advisory Board: David Cooper, University of New South Wales, Australia; Nikos Dedes, Positive Voice, Greece; Kevin Fenton, Public Health England, USA; David Pizzuti, Gilead Sciences, USA; Marco Vitoria, World Health Organization, Switzerland.

EuroCoord Secretariat: Kate Coughlin, University College London, United Kingdom; Silvia Faggion, Fondazione PENTA, Italy; Lorraine Fradette, University College London, United Kingdom; Richard Frost, MRC Regional Centre London, United Kingdom; Dorthe Raben, Københavns Universitet, Denmark; Christine Schwimmer, University of Bordeaux II, France; Martin Scott, UCL European Research and Development Office, United Kingdom. 